patient deteriorated in Group A and was withdrawn. The use of accessory muscles of respiration (AMR) reduced significantly in group $\mathrm{B}$ at 3,6 and $7 \mathrm{~h}(\mathrm{p}<0.01), \mathrm{BORG}$ dyspnoea score improved significantly $(p<0.01)$ in group $B$ after $1 \mathrm{~h}$. There was no difference between two groups in terms of improvement in RR, HR, FEV1 and ABG. In group B, the mean IPAP and EPAP used was $14.32 \pm 0.945$ and $7.16 \pm 0.472 \mathrm{~cm}$ of water, respectively.

Conclusion The use of NIPPV in patients with acute severe asthma though found to be useful in terms of faster resolution of dyspnoea and decrease in use of AMR but did not improve pulmonary functions significantly.

\section{S69 HOT HMV UK: PREVALENCE OF PERSISTENT SIGNIFICANT HYPERCAPNIA FOLLOWING ACUTE EXACERBATION OF COPD (AECOPD) REQUIRING NON-INVASIVE VENTILATION (NIV)}

doi:10.1136/thx.2010.150938.20

${ }^{1} \mathrm{P}$ Murphy, ${ }^{2} \mathrm{G} J$ Gibson, ${ }^{3} \mathrm{M}$ I Polkey, ${ }^{4} \mathrm{~N}$ Hart. ${ }^{1}$ Lane Fox Respiratory Unit, Guy's \& St Thomas' NHS Foundation Trust, London, UK; ${ }^{2}$ Department of Respiratory Medicine, Freeman Hospital, Newcastle-upon-Tyne, UK; ${ }^{3}$ Sleep \& Ventilation Unit, Royal Brompton \& Harefield NHS Foundation Trust, London, UK; ${ }^{4}$ Guy's \& St Thomas' NHS Foundation Trust and Kings College London NIHR Biomedical Research Centre, London, UK

Introduction Acute NIV is now standard therapy for hypercapnic respiratory failure (AHRF) complicating AECOPD. However, although chronic hypercapnia is an acknowledged poor prognostic factor in COPD and some data suggest a survival advantage with domiciliary NIV compared with oxygen alone, its use for chronic hypercapnic respiratory failure (CHRF) remains controversial. ${ }^{1}$ Patients with CHRF who have recently been treated acutely with NIV for AECOPD may be a particularly appropriate group in whom to consider long term domiciliary NIV.

Method We are conducting an RCT (HOT HMV UK) of domiciliary NIV plus oxygen against domiciliary oxygen alone in CHRF (defined as $\mathrm{PaCO}_{2}>7 \mathrm{kPa}$ ), focussing on patients who have recently received acute NIV for AECOPD, and we have evaluated the likely uptake of such treatment after resolution of the acute episode. All patients seen over a 5-month period at two tertiary centres for consideration of domiciliary NIV following acute NIV were assessed at least 2 weeks following resolution of AHRF and arterial blood gas analysis was performed.

Results 38 patients received acute NIV for an AECOPD. Mean $( \pm \mathrm{SD})$ age was $69 \pm 17$ years and $50 \%$ were male, with $\mathrm{PaCO}_{2}$ of $9.3 \pm 2.4 \mathrm{kPa}$ at acute presentation. Eight (21\%) patients died prior to review (six during initial admission, two shortly following discharge); four patients whose diagnosis was clarified as an overlap of obstructive sleep apnoea and COPD were treated on clinical grounds with NIV and one patient was already receiving domiciliary NIV. Of the remaining 25 patients, nine had $\mathrm{PaCO}_{2}<7 \mathrm{kPa}$ at 2 weeks, leaving $16(42 \%)$ with CHRF at 2 weeks post recovery. Importantly, seven (44\%) of these did not wish to be considered for a clinical trial involving domiciliary NIV, the principal reason being poor tolerance during the acute episode.

Conclusion More than $40 \%$ of patients requiring NIV for AHRF complicating AECOPD had CHRF with a $\mathrm{PaCO}_{2}>7 \mathrm{kPa}$, when assessed 2 weeks after discontinuation of acute NIV. However, nearly half of these reported negative experiences of acute NIV that made them reluctant to consider long term treatment.

\section{REFERENCE}

1. Elliott MW. Domiciliary non-invasive ventilation in stable COPD? Thorax 2009; 64:553-6.

\section{S70 NON-INVASIVE VENTILATION (NIV) IN CHRONIC OBSTRUCTIVE PULMONARY DISEASE (COPD) EXACERBATIONS WITH ACUTE HYPERCAPNIC RESPIRATORY FAILURE (AHRF) WITH PH}

doi:10.1136/thx.2010.150938.21

${ }^{1} \mathrm{~A}$ Thomas, ${ }^{1} \mathrm{~B}$ Beauchamp, ${ }^{2} \mathrm{~B}$ Chakraborty, ${ }^{1} \mathrm{E}$ Gallagher, ${ }^{1} \mathrm{~A}$ Ali, ${ }^{1} \mathrm{R}$ Mukherjee, ${ }^{1} \mathrm{D}$ Banerjee. ${ }^{1}$ Heart of England NHS Foundation Trust, Birmingham, UK; ${ }^{2}$ School of Mathematics, University of Birmingham, Birmingham, UK

Introduction Recent British guidelines on NIV suggest patients with AHRF and $\mathrm{pH}<7.26$ on arterial blood gases (ABG) should be managed by critical care (ITU) depending upon local circumstances with a low threshold for intubation, unless NIV is deemed to be the ceiling of treatment. The $7.26 \mathrm{pH}$ cut-off was derived from subgroup analysis of in-hospital mortality assessment from the study by Plant et al (Lancet 2000; 355:1931-5). The use of NIV as an alternative to endotracheal intubation in more severely acidotic COPD patients $(\mathrm{pH}<7.26)$ has been controversial but there is increasing evidence that the outcomes in such patients may not be any worse if treated with NIV.

Methods Analysis of initial ABG (pre-commencement of NIV) for AHRF secondary to COPD between 1 August 2004 and 31 December 2009 was performed. NIV was undertaken in a dedicated unit on a respiratory ward. The admission episodes were stratified by initial $\mathrm{pH}$ ranges (predictor variable) and in-hospital mortality was recorded (outcome variable).

Results Out of 728 (505 unique patients) admissions with COPD requiring NIV for AHRF, 282 admissions had a $\mathrm{pH}<7.26$. Of these, 224 admissions survived to discharge (mortality 20.6\%). Stratifying the admissions by $\mathrm{pH}$ categories of 0.05 , there is no significant difference in the mortality with $\mathrm{pH}$ ranges $7.2-7.25$ and $7.25-7.30$ ( $\mathrm{p}$ value $=0.845$ ). If all COPD admissions requiring NIV $(n=728)$ are stratified into two groups above and below 7.2, the ratio of odds of survival below pH 7.2 drops sharply ( $p=0.000000088)$ : Abstract S70 Table 1 .

Abstract S70 Table

\begin{tabular}{llll}
\hline $\mathbf{p H}$ & Survived & Not Survived & $\mathbf{n}$ \\
\hline$<7.2$ & $110(73.3 \%)$ & $40(26.7 \%)$ & 150 \\
$\geq 7.2$ & $517(89.4 \%)$ & $61(10.6 \%)$ & 578 \\
Total & 627 & 101 & 728 \\
\hline
\end{tabular}

Conclusion Mortality in the group with pH 7.20-7.25 was similar to the group with $\mathrm{pH} 7.26-7.30$, suggesting that NIV on a dedicated respiratory ward can manage COPD patients with AHRF and $\mathrm{pH}$ $<7.26$. Our findings suggest that it is probably justified to recommend that the initial $\mathrm{pH}$ cut-off is modified from $\mathrm{pH}>7.26$ to $\mathrm{pH}$ $>7.20$ for ward-based NIV in COPD exacerbations with AHRF. This has important resource implications, given that more patients could be managed in a ward-based dedicated NIV unit rather than the utilising ITU beds in the UK National Health Service.

\section{Novel outcomes and interventions in pulmonary rehabilitation
S71 AMBULATORY OXYGEN IMPROVES THE EFFECTIVENESS OF PULMONARY REHABILITATION (PR) IN SELECTED PATIENTS

doi:10.1136/thx.2010.150938.22

F J Dyer. Surrey Community Health, Chertsey, UK

Rationale The acute effect of supplemental oxygen on exertion is well documented although its use in PR has not yet been clearly 
established. Two small studies have previously shown no benefit; this may have been due to study power or methodology. Our study investigated whether ambulatory oxygen provides additional benefit to patients undergoing PR who meet the 2006 UK Department of Health criteria for ambulatory oxygen use.

Methods A single blind (researcher) randomised controlled trial compared the effect of a 6 week PR programme either with or without ambulatory oxygen. The study was powered to show an $80 \%$ difference between groups. Eligible patients were those who desaturated on baseline exercise testing by $>4 \%$ to $<90 \%$ and whose exercise tolerance improved by $>10 \%$ with ambulatory oxygen. Outcome measures included the Endurance Shuttle Walk Test (ESWT) and the self report Chronic Respiratory Questionnaire (CRQ-SR).

Results Between September 2007 and June 200962 patients consented; one patient withdrew choosing to use ambulatory oxygen, ten dropped out of PR. The majority of subjects had Chronic Obstructive Pulmonary Disease; eight had another chronic respiratory condition. Groups were similar at baseline except for weight and BMI (higher in the room air (RA) group). A far greater improvement in ESWT was found in the oxygen group $(p=0.000)$ (Abstract S71 Table 1). When the acute effect of oxygen is excluded the oxygen group improved by $75 \%$ more than the RA group; this did not meet statistical significance since the study was powered to show an $80 \%$ improvement. The oxygen group gained improvements in three CRQ-SR domains (emotion, fatigue and mastery) above the minimally clinically important difference (MCID) but this was not the case for the RA group; the difference between groups for these domains also reached the MCID. Improvements in the dyspnoea domain were similar between groups, although the oxygen group walked $490 \mathrm{~m}$ (122\%) further.

\section{Abstract S71 Table 1 ESWT outcome}

\begin{tabular}{lcclll}
\hline & RA group & $\mathbf{0}_{2}$ group & Difference & 95\% Cl & p \\
\hline Mean change m (SD) & $401(391)$ & $891(477)$ & 490 & 245 to 735 & $0.000^{*}$ \\
Mean change \% (SD) & $77(76)$ & $199(214)$ & 122 & 32 to 211 & $0.009^{*}$ \\
Mean change secs (SD) & $380(358)$ & $682(311)$ & 302 & 113 to 491 & $0.002^{*}$ \\
\hline
\end{tabular}

*Unpaired $t$-test.

Conclusion For patients who desaturate and with an acute positive response to oxygen, ambulatory oxygen significantly enhances the effect of PR.

Funding This study was supported by a $£ 15000$ British Lung Foundation grant.

\section{S72 THE UTILITY OF THE MODIFIED BODE INDEX (INCORPORATING THE INCREMENTAL SHUTTLE WALKING TEST) IN ASSESSING SURVIVAL IN PATIENTS WITH COPD SCREENED FOR PULMONARY REHABILITATION (PR)}

doi:10.1136/thx.2010.150938.23

J E A Williams, V Warrington, S J Singh, R Green. Pulmonary Rehabilitation Research Group, University Hospitals of Leicester NHS Trust, Leicester, UK

Background We have previously reported that distance walked on the Incremental shuttle walking test (ISWT) can be substituted for the 6 min walking distance (6MWD) as the exercise ("E" component) of the BODE index (ERS 2009). This study examines 5 year survival in a cohort of patients with COPD and examines the validity of the modified BODE index as an independent predictor of survival in COPD.

Method Hospital records of 1127 patients with COPD referred to PR (mean (SD) $\mathrm{FEV}_{1} 46.1$ (16.2)\% predicted, age 68.4 (9.0) years) were examined to assess 5 -year survival. A multivariate cox proportional

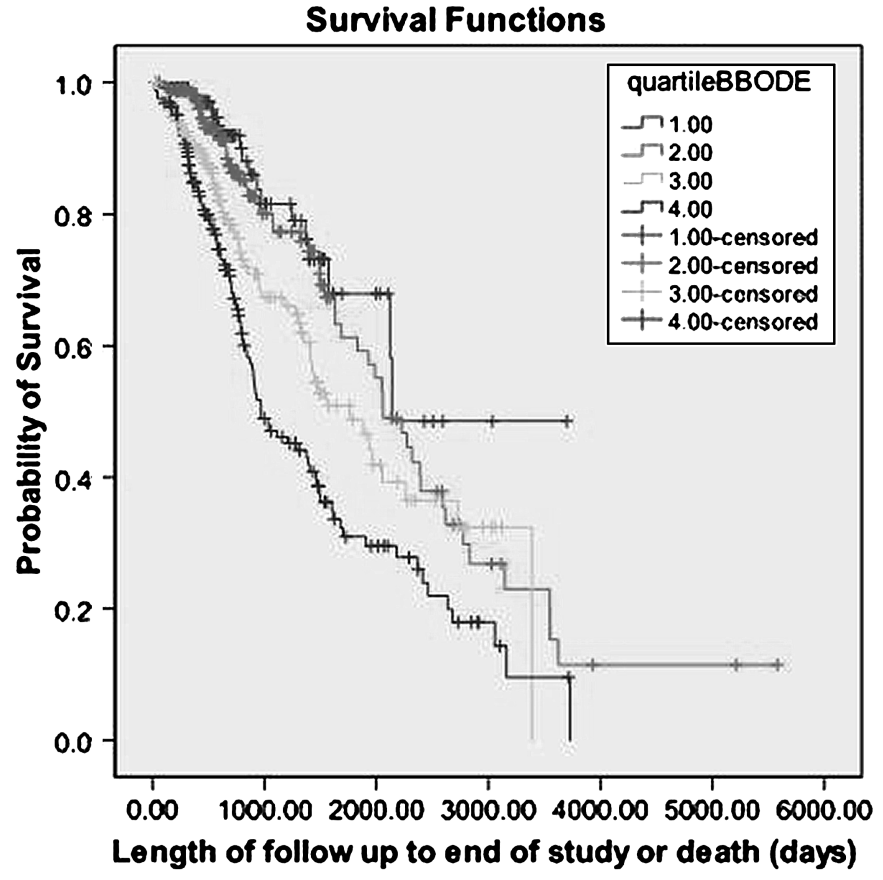

Abstract S72 Figure 1 Kaplan-Meier survival curves for the four quartiles of the BODE index.

hazards model including the variables gender, age, pack years, $\mathrm{FEV}_{1}$, FVC, BMI, MRC dyspnoea score and ISWT was used to identify independent factors predicting all cause mortality. A BODE score was then assigned to 626 patients with sufficient data for analysis. Cut-off points for the ISWT quartiles were as follows: $<80 \mathrm{~m}=3$, $80-149 \mathrm{~m}=2,150-249 \mathrm{~m}=1,>250 \mathrm{~m}=0$. Cox regression was used to predict hazard ratios for the modified BODE index, adjusted for the independent prognostic factors identified by initial analysis Kaplan-Meier analysis of survival by quartile of the BODE index was then performed and compared with the log rank test.

Results Overall 5 -year survival was $41.9 \%$. Three of the four factors in the BODE index (BMI, exercise capacity, and MRC dyspnoea) were statistically significant independent predictors of survival whilst the fourth, $\mathrm{FEV}_{1} \%$ predicted was of borderline significance $(p=0.08)$. In addition age and pack years smoked were also independent prognostic factors. The mean (SD) BODE index was significantly higher in patients who had died at 5 years $(5.9(2.2)$ ) than those that had survived (4.8(2.2)), $\mathrm{p}<0.0001$. The modified BODE index was a significant predictor of death even corrected for age and pack years smoked (adjusted hazard ratio (95\% CI) 1.26 (1.17 to 1.35), $\mathrm{p}<0.0001$ ). Kaplan-Meier survival analysis confirmed that each quartile increase in the BODE index was associated with increased mortality $(p<0.0001)$ (Abstract S72 Figure 1).

Conclusion The BODE index using the ISWT as the exercise component, is a useful tool for predicting survival in patients with COPD.

\section{S73 4-METRE GAIT SPEED AS A FUNCTIONAL OUTCOME MEASURE IN PATIENTS WITH CHRONIC OBSTRUCTIVE PULMONARY DISEASE (COPD)}

doi:10.1136/thx.2010.150938.24

A V Donaldson, B E Garfield, M S Patel, A L Clark, M I Polkey, W D Man. Biomedical Research Unit and Harefield Pulmonary Rehabilitation Team, Royal Brompton \& Harefield NHS Foundation Trust, London, UK

Introduction Well-established functional outcome measures in COPD include the 6-min walk test (6MW) and incremental shuttle 